\title{
The carpal tunnel syndrome in hypertensive patients treated with beta-blockers
}

\author{
M.K. Emara and A.M. Saadah \\ Department of Medicine, Mubarak Al Kabeer Hospital, Kuwait.
}

\begin{abstract}
Summary: We report the development of the carpal tunnel syndrome in 3 hypertensive men (aged 46-58) treated with beta-blockers (propranolol in two patients and metoprolol in the 3rd) for 8-11 years. Known causes of carpal tunnel syndrome were excluded in each case. Withdrawal of the betablocker was followed by complete resolution of symptoms and signs after a period of 8-10 weeks. No evidence of recurrence could be detected during follow-up of 7-18 months. The possible mechanisms of drug-induced carpal tunnel syndrome are briefly discussed.
\end{abstract}

\section{Introduction}

Adverse effects due to beta-blockers may be predictable according to their known pharmacological properties or may be unpredictable. The latter include the oculo-muco-cutaneous syndrome with practolol and the high titres of antinuclear and other auto-antibodies and LE cells with practolol and acebutolol. ${ }^{1}$ Although parasthesiae as well as nerve deafness were mentioned among the side effects, ${ }^{2}$ to the best of our knowledge, there has been no report of the carpal tunnel syndrome (CTS).

We report here an association between prolonged beta-blocker therapy and the occurrence of CTS in three hypertensive men.

\section{Patients}

The first patient, aged 58, had been treated with propranolol $120 \mathrm{mg} /$ day for more than 8 years. He presented with symptoms and signs of bilateral CTS, more on the right side, confirmed by neurological and electrodiagnostic evaluation. While on vacation abroad his drug supply ran out. After about 6 weeks without treatment, he noted that the burning sensation in his hands gradually decreased,

Correspondence: M.K. Emara, M.B., D.M., D.C.V.D., F.R.C.P. (Ed.), F.R.C.P., Department of Medicine, Faculty of Medicine, Kuwait University, P.O. Box 24923, Safat - 13110, Kuwait.

Accepted: 4 November 1987 and later completely disappeared. When examined at that stage, no abnormality could be detected and median nerve conduction studies (distal motor and sensory latencies) were within normal limits.

The second case, a man aged 46, had been treated with propranolol $120 \mathrm{mg} /$ day, chlorthalidone $50 \mathrm{mg} / \mathrm{day}$, and supplementary potassium for more than 11 years. The third patient, aged 52, also had coronary disease and had been treated with metoprolol $200 \mathrm{mg}$ /day for more than 9 years, while isosorbide dinitrate $40 \mathrm{mg} /$ day and nifedipine $30 \mathrm{mg} /$ day were added for the last 2 years. Both patients presented with sensory symptoms and signs characteristic of CTS in the right hand, including sensory deficit, positive wrist flexion test and impaired median nerve conduction studies. Symptoms had been present for about 4 months in Case 2 and 3 months in Case 3. There were no motor symptoms or signs and the left hand was normal in both patients.

There was no history suggestive of occupational or accidental trauma. Clinical, laboratory and radiological investigations (including cervical spine, both wrists and hands) were within normal ranges. There was no evidence of peripheral neuropathy or any of the known causes of CTS. The beta-blocker was withdrawn. Gradual improvement, followed by complete relief, was reported after 8 weeks (Case 2) and 10 weeks (Case 3). This was confirmed by resolution of the sensory deficit in the hand, negative wrist flexion test and within normal median nerve conduction velocities. During followup for 18 months (Case 1), 13 months (Case 2) and

(C) The Fellowship of Postgraduate Medicine, 1988 
7 months (Case 3), the patients have been free of any symptoms or signs suggestive of recurrence. Control of blood pressure during follow-up was maintained by methyldopa $500 \mathrm{mg} /$ day.

\section{Discussion}

CTS may result from any factor leading to deformity in the size of the carpal tunnel, or swelling of its contents. The median nerve may be injured by direct mechanical pressure, or its function may be impaired by venous congestion, oedema and arterial insufficiency. The important contribution of the latter changes explains the rapid functional recovery which occurs hours after decompression by surgery or local steroid injections. $^{3}$ The usual causes of CTS include pregnancy, diabetes, myxoedema, acromegaly, myeloma, rheumatoid arthritis and trauma. ${ }^{3}$ It may also be idiopathic in more than $50 \%$ of cases, mainly premenopausal women. ${ }^{4}$

A few drugs have been suspected of causing it by different mechanisms. ${ }^{5}$ Oral contraceptives, danazol and progesterone may act by leading to oedema as a result of fluid retention, thalidomide and disulfiram act by producing a peripheral neuropathy. ${ }^{5}$ CTS had been associated with pyridoxine deficiency, which when given in large doses produced marked improvement with avoidance of surgery in some cases. ${ }^{6}$ Pyridoxine deficiency might lead to CTS not only by producing peripheral neuropathy, but also by leading to synovial subendothelial oedema with proliferative and fibrotic changes. ${ }^{7}$ The andidepressant tranyl-

\section{References}

1. Cruickshank, J.M. How safe are beta-blockers in the 1980's, Symposium on beta-blockers, 1983. In: Robertson, J., Kaplan, N., Coldwell, H., Speight, F. (eds) 25 Drugs (Suppl. 2), 331-340.

2. Reynolds, E. \& Prasad, A. Propranolol and other beta-adrenergic blocking agents. In Martindale The Extra Pharmacopaeia, 28th edition. The Pharmaceutical Press, London, 1982, p 1324.

3. Dorwart, B. Carpal tunnel syndrome, a Review. Semin Arthritis Rheum, 1984, 14: 134-140.

4. Dekel, S., Papaioannon, T., Rushworth, G. \& Coates, R. Idiopathic carpal tunnel syndrome caused by carpal stenosis. Br Med J 1980, 280: 1297-1303.

5. Castle, W.M. Drugs and fibrotic reactions, adverse reaction group. I.C.I. Adverse Drug Reaction Bulletin 1985, 113: 420-423.

6. Ellis, J., Folkers, K., Levy, M. et al. Response of vit $B_{6}$ deficiency and the carpal tunnel syndrome to cypromine was thought to lead to the syndrome by impairing pyridoxine metabolism. ${ }^{8}$ The association of CTS with pyridoxine deficiency was, however, questioned in other studies. ${ }^{9}$

The relationship between prolonged beta-blocker therapy and the development of CTS is suggested by the disappearance of all symptoms and signs after withdrawal of the drug. The mechanism of this association is not clear to us. It may result from fluid retention and oedema secondary to betablocker therapy. ${ }^{2}$ The presence of a latent peripheral neuropathy, or of proximal compression might make the nerve vulnerable to lesser degrees of pressure at the wrist 'double crush'. ${ }^{10}$ There was no evidence of any of these complications in our patients. Pyridoxine deficiency however could not be excluded because the appropriate laboratory tests were not available.

Beta-blockers may lead to peripheral vasospasm and an association has been reported between this and CTS, but the vasospasm was thought to be due to pressure over the autonomic fibres in the nerve. ${ }^{11}$ Moreover none of our patients had any symptoms or signs suggestive of peripheral vasospasm. We believe that this association needs further documentation. All our patients on long term beta-blocker therapy are being asked now about symptoms suggestive of CTS, in a more extensive study.

\section{Acknowledgement}

We would like to thank Mrs Aloma D'Souza for secretarial assistance.

pyridoxine. Proc Natl Acad Sci, USA 1982, 79: 7494 7498.

7. Ellis, J., Folkers, K., Watanabe, T. et al. Clinical results of a cross-over treatment with pyridoxine and placebo of the carpal tunnel syndrome. Am J Clin Nutr 1979, 132: 2040-2046.

8. Harrison, S., Stewart, J., Lovelace, R. \& Quitkin, F. Case report of carpal tunnel syndrome associated with tranylcypromine. Am J Psychiatry 1983, 140: 12291230.

9. Amadio, P. Pyridoxine as an adjunct in the treatment of carpal tunnel syndrome. J Hand Surg (Am) 1985, 10A: 237-241.

10. Upton, A.R. \& McComas, H.J. The double crush in nerve entrapment syndrome. Lancet 1973, ii: 359-362.

11. Waller, D. \& Danathan, R. Raynaud's syndrome and carpal tunnel syndrome. Postgrad Med J 1985, 61: 6162. 